\title{
Utilización de la plataforma de comunicación Lingalog para la interacción de dos clases de grado sexto en Francia y Colombia mediante la resolución de problemas con el uso de un lenguaje matemático ${ }^{1}$
}

\author{
Using Lingalog communication platform for the interaction \\ of two sixth grade classes in France and Colombia by \\ solving problems using mathematical language
}

\begin{abstract}
Usando a plataforma de comunicação Lingalog para a interação de duas turmas de sexta na França e na Colômbia por resolução de problemas utilizando a linguagem matemática
\end{abstract}

Recibido: mayo de 2013

Aceptado: agosto de 2013
Andrea Carolina Dávila Lozano ${ }^{2}$

\begin{abstract}
Resumen
Actualmente, gracias a internet y a las nuevas tecnologías, es posible realizar diferentes estudios, intercambios e innovaciones a nivel educativo. Un ejemplo es la creación y utilización de plataformas de comunicación en el estudio de lenguas como Lingalog (plataforma Francesa), donde se llevó a cabo una experiencia de intercambio entre estudiantes de secundaria de Francia y Colombia. Partiendo de esta experiencia, se consideró la posibilidad de ampliar el uso de Lingalog en función del aprendizaje de las matemáticas. Esto con el fin de aprovechar el interés que conlleva el intercambio entre clases, de dos países diferentes, para trabajar en la resolución de problemas e identificar el uso de un lenguaje matemático como medio de comunicación. De esta manera, se escogieron clases de grado sexto pertenecientes a colegios públicos ubicados en zonas vulnerables en las ciudades de Lyon (Francia) y de Bogotá (Colombia). Tomando como referencia las similitudes encontradas en los programas de ambos países y gracias a la colaboración de los profesores de matemáticas en cada país, se logró crear un proyecto franco-colombiano en un espacio que se llamo Mathice dentro de la plataforma de comunicación Lingalog. Para este intercambio, se propone un trabajo a partir de la resolución de problemas, haciendo uso de les narrations de recherche. ${ }^{3}$
\end{abstract}

Palabras clave: TIC; Otras nociones de Educación Matemática; resolución de problemas; resolución de situaciones problema; les narrations de recherche; lenguaje matemático; aula; recursos didácticos; recursos informáticos; internet; plataforma de comunicación.

\footnotetext{
1 Artículo de Investigación.

2 Universidad Pedagógica Experimental Libertador. Venezuela. Contacto: jommadail@gmail.com

3 El término «Les narration de recherche» corresponde a un tipo de situaciones problema utilizadas en Francia con el fin de que los estudiantes realicen una producción escrita, partiendo del uso de un lenguaje natural en la búsqueda de soluciones a problemas matemáticos y haciendo uso de diferentes herramientas de la plataforma como la mensajería personal, la wiki el chat y el fórum para compartir su trabajo con estudiantes de otro país.
} 


\section{Abstract}

Today, thanks to the Internet and new technologies, you can perform various studies, exchanges and innovations to educational level. One example is the creation and use of communication platforms in the study of languages as Lingalog (French platform), which held an exchange experience among high school students in France and Colombia. Based on this experience, we considered the possibility of extending the use of Lingalog depending on the learning of mathematics. This is to take advantage of the interest involved the exchange between classes, in two different countries, to work on solving problems and identify the use of mathematical language as a means of communication. Thus, were chosen sixth grade classes belonging to public schools located in vulnerable areas in the cities of Lyon (France) and Bogota (Colombia). Drawing on the similarities found in the programs of both countries and thanks to the collaboration of mathematics teachers in each country, it was possible to create a Franco-Colombian in a space that was called Mathice within Lingalog communication platform. For this exchange, we propose a job from solving problems, making use of their narrations of recherche.

Keywords: ICT, Mathematics Education Other concepts, problem solving, resolution of problem situations; them de recherche narrations, mathematical language; classroom teaching resources, computing resources, Internet, communication platform.

\section{Resumo}

Hoje, graças à Internet e às novas tecnologias, é possível realizar vários estudos, intercâmbios e inovações ao nível de escolaridade. Um exemplo é a criação e utilização de plataformas de comunicação no estudo de línguas como Lingalog (plataforma francês), que realizou uma troca de experiências entre os estudantes do ensino médio na França e na Colômbia. Com base nesta experiência, consideramos a possibilidade de estender o uso de Lingalog dependendo da aprendizagem da matemática. Este é tirar vantagem do interesse envolveu a troca entre classes, em dois países diferentes, para trabalhar em resolver os problemas e identificar o uso de linguagem matemática, como meio de comunicação. Assim, foram escolhidos sexta turmas pertencentes às escolas públicas localizadas em áreas vulneráveis nas cidades de Lyon (França) e Bogotá (Colômbia). Com base nas semelhanças encontradas nos programas de ambos os países e, graças à colaboração de professores de matemática em cada país, foi possível criar um franco-colombiana em um espaço que foi chamado Mathice dentro plataforma de comunicação Lingalog. Para essa troca, propomos um trabalho de resolução de problemas, fazendo uso de suas narrações de recherche.

Palavras-chave: TIC, Educação Matemática Outros conceitos, resolução de problemas, resolução de situações-problema;-los de Recherche narrações, linguagem matemática, recursos de ensino em sala de aula, os recursos de computação, Internet, plataforma de comunicação. 


\section{Presentación}

Lingalog ${ }^{4}$ es una plataforma de comunicación creada que nace en el 2005 en el centro de lenguas de la universidad Lumière Lyon 2, destinada al aprendizaje de las lenguas en autonomía solidaria y que ofrece un espacio a proyectos pedagógicos, con el objetivo de promover el desarrollo de competencias plurilingües. La idea de realizar un intercambio entre clases de estos dos países nace por dos razones: la primera surge del encuentro con el administrador de la plataforma Jean-Pierre Chavagne, luego de colaborar en un proyecto franco-colombiano en lenguas realizado en la plataforma para una investigación de máster en ciencias de la educación; la segunda surge de la ausencia de registros escritos de una experiencia o trabajo como éste en educación matemática a nivel escolar. Por esta razón, se decidió explorar esta experiencia innovadora en educación matemática y así ampliar el uso de la plataforma. Para ello, se contó inicialmente con el interés y la colaboración de dos profesores de matemáticas, uno en cada país para trabajar en este proyecto franco-colombiano, con quienes se escogieron dos clases de grado sexto, una en Francia y otra en Colombia, ambas pertenecientes a colegios públicos ubicados en zonas vulnerables.

En este estudio se busca principalmente mostrar cómo se pueden realizar intercambios entre estudiantes de diferentes países por medio de la plataforma Lingalog y motivarlos en el aprendizaje de las matemáticas. Además, trabajando en la resolución de problemas y particularmente les narrations de recherche, se quiere identificar la utilización de un lenguaje matemático para comunicar las ideas, Beyer citado por (Serrano, 2002) define el lenguaje matemático "como el código empleado por una persona para transmitirle a otra(s) persona(s) ideas matemáticas" p.2. Por consiguiente, se escogieron algunas situaciones de "les narrations de recherche", que responden al trabajo propuesto a los profesores dentro del programa de matemáticas en secundaria. Estas tienen el propósito de buscar que el estudiante narre todas las ideas y procedimientos que tiene en mente para encontrar la solución del enunciado de una narración. Los enunciados son específicos y conllevan una serie de tareas de parte del estudiante y del profesor para lograr que el estudiante pierda el miedo al error y lo conciba como un elemento más de la búsqueda. Ésta es una manera de iniciar al estudiante en un razonamiento de tipo investigativo.

Finalmente, otro objetivo es promover las actitudes colaboración y diálogo, reduciendo las actitudes individualistas y de pasividad. Al respecto, Gómez (2002) afirma "Diferentes estudios han mostrado el importante papel que juegan las interacciones sociales como facilitadoras del desarrollo cognitivo desde los años setenta [Doise et al, 1975], [Doise et al, 1976], [Doise, Mugny, 1981]"p.5. Así, Gómez y Carrillo (2009) encontraron que a partir de experiencias colaborativas:

(...) podemos decir que ningún grupo de alumnos dejo sin intentar resolver el ejercicio, esto es, el 'abandono casi inmediato' de un problema que me cueste resolver se redujo notablemente (...) Esto constituye un claro indicador del interés que este tipo de entorno puede aportar a la resolución de problemas.

Los alumnos ganan en capacidad de generar conjeturas y argumentaciones, porque la interacción electrónica les ayuda a perder el miedo de explicar sus razonamientos y a desdramatizar los errores, que pasan a formar parte de los pasos que pueden ocurrir durante el proceso de aprendizaje. Se puede decir incluso que hacen más persistentes sus tareas. (Gómez \& Carrillo, 2009, p. 9)

En consecuencia, al culminar este trabajo investigativo, se pretende responder independientemente del contexto y de la lengua de los estudiantes, ¿Cómo a través de la plataforma Lingalog se puede motivar a los estudiantes en el aprendizaje y la resolución de problemas en matemáticas y a su vez fomentar el uso de un lenguaje matemático?

Es importante considerar que la utilización de una plataforma de comunicación como Lingalog

4 http://lingalog.net/dokuwiki/:es:accueil 
se puede ver como una alternativa innovadora y diferente a los métodos tradicionales de enseñanza y aprendizaje en matemáticas, en ese sentido aportando a la investigación en didáctica de las matemáticas. De igual manera, se podrían predeterminar las condiciones para realizar intercambios bilingües a nivel educativo en matemáticas utilizando nuevas tecnologías de comunicación, y así crear proyectos que contribuyan al aprendizaje de las matemáticas mediante la resolución de problemas superando los límites de la distancia y la lengua.

\section{Marco de referencia conceptual}

Inicialmente, uno de los trabajos que motivó esta investigación fue la tesis doctoral de Gómez (2002) de la cual, para la presente investigación, se retoma el hecho de utilizar preferiblemente preguntas abiertas con diferentes opciones de respuesta o con una única respuesta, pero con diferentes estrategias de solución. Además, se seleccionó uno de los problemas propuestos en el trabajo de dicho autor para plantearlo a los estudiantes en Lingalog.

También, para el diseño y planeación, se basó en algunos de los resultados obtenidos de un estudio realizado por Dumont (2009) al entorno Lingalog, a nivel ergonómico y en el aprendizaje de las lenguas. A partir de éste, se retomó de las conclusiones, que son pocos los estudiantes que trabajan fuera de las horas de curso en las actividades propuestas en la plataforma, aunque, el autor afirma que los estudiantes encontraban este tipo de intercambios motivantes e interesantes para el aprendizaje de las lenguas. Por esta razón, se pensó en enfocar el trabajo solamente en las horas de clase de matemáticas.
Para la selección de los otros enunciados que harían parte actividades propuestas a los estudiantes, se tomaron dos libros sobre "les narrations de recherche" Artigue \& Winslow (2010) y Bonafe (2002), donde se presentan no sólo los problemas, sino también los resultados de las experiencias, y un análisis y caracterización de este tipo de problemas. A partir de estas experiencias y de la metodología de trabajo que se propone utilizar en clase, se pudo pensar en cómo adaptar el trabajo de los estudiantes incluyendo la participación en la plataforma Lingalog, para así organizarlo antes del comienzo de la exploración.

Existen otros proyectos como Math-Bridge ${ }^{6}$ y como VMT $^{7}$ Projet (the Virtual Math Teams Projet), que se enfocan en el aprendizaje de las matemáticas y que a nivel informático son más complejos. Por lo tanto, cuentan con más herramientas a nivel matemático que Lingalog. Aun así, se ha centrado la atención en VMT Project dado que, por una parte, la documentación encontrada al respecto puede aportar al análisis de resultados del presente estudio y por otra parte, es un proyecto que se asemeja más al tipo de trabajo que se busca con Lingalog. También se encontraron algunos documentos en Colombia (Cogollo) y (Rodriguez), que dejan ver un interés por los proyectos en el aula para el aprendizaje de las matemáticas, haciendo uso de plataformas como WizIQ $^{8}$ y redes sociales como Facebook, Messenger, entre otras. La única variación es que las interacciones entre los estudiantes en el VMT Projet y los proyectos en Colombia, son en una misma lengua (inglés) o (español) y que, a diferencia de Lingalog, el intercambio se hace en dos lenguas (francés y español), lo cual podría limitar tanto la comunicación como el intercambio de ideas.

5 Las "narraciones de recherche" requieren un tiempo considerable de trabajo entre 3 o 4 horas de clase.

6 Una de las primeras plataformas en línea e-Learning, Europeas. Diseñada y desarrolladla a partir del estudio de nueve universidades de siete países. Esta plataforma brinda cursos de matemáticas en línea, ofrece cientos de herramientas para el aprendizaje matemático en siete lenguas, en la cual pueden participar e interactuar profesores y estudiantes. http://www.math-bridge.org/

7 Es un proyecto que tiene como objetivo catalizar y nutrir los "networks"y discusiones en matemáticas "online". Lo que se hace por medio de chats en pequeños grupos de estudiantes. La visión es que personas de todas partes del mundo logren conversar con otros sobre diferentes temas en matemáticas de interés común y gradualmente crear una comunidad virtual con un discurso en matemáticas.

8 Education.Online http://www.wiziq.com/ 


\section{Metodología}

Como metodología de investigación se ha escogido la ingeniería didáctica. Se le denomina ingeniería didáctica, a una forma de trabajo didáctico similar al trabajo de un ingeniero, quien para realizar un proyecto determinado, toma como base los conocimientos científicos de su dominio y acepta someterse a un control de tipo científico, pero que, a su vez, se ve obligado a trabajar con objetos mucho más complejos que los objetos depurados por la ciencia (Artigue, 1995). Luego, como lo indica la ingeniería didáctica, el proceso experimental de la investigación se divide en 4 fases: la fase uno (el análisis preliminar), la fase dos (la concepción y análisis a priori de las situaciones didácticas), la fase tres (la experimentación) y para terminar la fase cuatro (el análisis a posteriori y evaluación).

En la primera fase, para el análisis preliminar se tomaron todos los documentos oficiales a nivel educativo en matemáticas de cada país para hacer un estudio de cada población y, al mismo tiempo, se realizó un análisis comparativo entre ambos sistemas educativos. Para ello, se han utilizado documentos como Artigue \& Winslow (2010) para realizar un "approche structuraliste", del cual retomamos los niveles de comparación propuestos por la TAD (Teoría Antropológica de la Didáctica). Todo esto fue de gran utilidad para comparar el contexto institucional en Francia y en Colombia, teniendo en cuenta los textos oficiales y el programa seguido por el profesor en cada institución.

En las fases dos y cuatro, con el objetivo de realizar el análisis de resultados, se tomó como referencia la metodología para analizar las actividades de los estudiantes en clase (Robert, 2008), en la cual se estudian las diferentes tareas realizadas por el profesor y el estudiante a través de un análisis a priori (de lo esperado) y un análisis a posteriori (de lo obtenido) de la experiencia realizada y de la utilización de la plataforma de comunicación.

La fase tres, se inició desde la selección de la población del trabajo, la formulación de objetivos y el establecimiento de las condiciones de la investigación. Durante la ejecución de esta fase, se dio lugar a la construcción del espacio en la plataforma Lingalog, la organización y coordinación del trabajo del proyecto franco-colombiano, el cual fue nombrado MATHICE para ubicarlo en el menú de proyectos de la plataforma. Luego de la creación, se procedió a la inscripción y familiarización de los profesores y estudiantes con herramientas tales como la mensajería, la wiki y el fórum de la plataforma Lingalog y se estableció el contrato didáctico, donde por ejemplo, el rol del profesor es el de guía y mediador frente al trabajo del estudiante.

Finalmente, para la recolección de los datos en las diferentes sesiones de trabajo, se contó con los registros escritos realizados en la plataforma (mensajería, wiki y fórum) de ambos países, la observación y los registros en video de algunas sesiones con los estudiantes de Francia y Colombia.

\section{Análisis de datos}

Como la ingeniería didáctica lo indica, el análisis de datos hace parte de la última fase de la investigación que es el análisis a posteriori y evaluación, la cual se basa en el conjunto de datos recolectados que, como se mencionó anteriormente, son todos los registros escritos en clase y sobre la plataforma, los videos y las observaciones realizadas en cada país. En esta parte, la aceptación o refutación de las hipótesis formuladas depende de la confrontación del análisis a priori y a posteriori. Vale la pena aclarar, que el análisis a posteriori de los datos de esta investigación sigue en curso y la totalidad de los resultados y conclusiones se presentaran más adelante. Aun así, luego de haber realizado un análisis a priori y habiendo terminado la experimentación se puede presentar un adelanto de algunos resultados encontrados.

En la concepción y análisis a priori de este trabajo no se había pensado cómo la diferencia de calendarios escolares y el retraso en el programa de matemáticas del año, afectaría la recolección de los datos. Por ejemplo, una de las consecuencias de ello es que solo se tendrán en cuenta para el análisis completo los resultados, una actividad de las tres propuestas, porque fue la única que realizaron 
los estudiantes de ambos países. También, en el análisis a priori no se consideró la posibilidad de que los estudiantes se motivaran por aprender una lengua extranjera ni se comunicar en ella. Aun así, los estudiantes en Francia al final de la experiencia, tuvieron como iniciativa realizar un video para los estudiantes en Colombia, donde cada uno saludaba y se presentaba, también uno de los estudiantes en Francia escribió por medio de la mensajería personal de Lingalog, un saludo y presentación a su compañero en Colombia en español. Por otro lado, durante la experiencia se pudo confirmar lo dicho por los estudios sobre el trabajo colaborativo, ya que se observó que todos los estudiantes presentes en la experiencia de una u otra manera encontraron una solución al problema.

\section{Conclusiones}

Las conclusiones aún no han sido establecidas puesto que el análisis a posteriori de los resultados no ha sido realizado completamente y es de gran importancia mostrar el contraste entre el análisis a priori y a posteriori del trabajo. No obstante, hasta este momento, tomando como base los diferentes documentos estudiados y luego de haber terminado la fase de experimentación, se puede decir que proyectos como éste son posibles en la medida en que los recursos informáticos proporcionen las herramientas necesarias para que los estudiantes expresen sus ideas.

\section{Referencias}

Adamson B. \& Morris P. (2010). Comparer des programmes d'études. En MASON M. (Dir). Recherche Comparative en éducation. Groupe De Boeck. Page 247.

Artigue M. (1995), Ingeniería didáctica. In ARTIGUE M., DOUADY R., MORENO L. y GOMEZ P. (Ed.) Ingeniería didáctica en educación matemática. Páginas 33 a la 58.
Grupo editorial Iberoamérica, Una empresa docente. Bogotá, Colombia.

Artigue M. \& Winslow C. (2010) Recherches en didactique des mathématiques. International Comparative Studies on Mathematics Education: a Viewpoint from the anthropological Theorie of didactics.

Bonafe F. (Coord.) (2002). Les narrations de recherche, de l'école primaire au lycée.

Cogollo, G. E. M. Desarrollo de aprendizaje significativo, mediante el uso adecuado de las redes sociales y las NTIC'S. Caso pedagógico "la clase virtual más larga del mundo". Bolívar, Colombia. [DOC] à partir de virtualeduca. info

Cori R. \& Delediq A. (2000-2001) (Dir.). Expériences de narration de recherche en mathématiques. IREM de Paris 7. ACL les éditions du Kangourou.

Gomez M. (2002). Estudio teórico, desarrollo, implementación y evaluación de un entorno de enseñanza colaborativa con soporte informático (CSCL) para matemáticas. Thèses doctoral de l'université Complutense de MADRID. Recuperado de : http://www. ucm.es/BUCM/tesis/edu/ucm-t26874.pdf

Gomez M. \& Carrillo R. (2009) La resolución de problemas Matematicos en entornos colaborativos con soporte tecnológico (TIC). Recuperado de: cimm.ucr.ac.cr/ojs/index. php/eudoxus/article/download/265/238.

MEN Ministerio de Educación Nacional. Serie lineamientos curriculares Matemáticas. Colombie. Recuperado de : http://www. mineducacion.gov.co/cvn/1665/articles-89869_archivo_pdf9.pdf

MEN Ministerio de educación Nacional. Estándares Básicos de Competencias en matemáticas. Colombie. Recuperado de: http://www.mineducacion.gov.co/1621/articles-116042_archivo_pdf2.pdf. 
MInistere de l'education nationale. B.O., Programmes du collègue, programmes de l'enseignement de mathématiques. Bulletin officiel spécial nº du 28 août 2008 . France.

Robert A. (2008).Une méthodologie pour analyser les activités (possibles) des élèves en classe. En VANDEBROUCK F (Coord). La classe de mathématiques activités des élèves et pratiques des enseignants. Pages 45 à 57. Editions OCTARES, Toulouse, France.

Rodríguez, P. B. Redes Sociales para la Enseñanza y el Aprendizaje de las Matemáticas. Experiencia MathClub Virtual. Guajira, Colombia. [DOC] à partir de virtualeduca. info
Serrano, W. G. (2002). El discurso matemático en el aula. Un análisis desde la observación del curso Sistemas Numéricos. Sapiens. Revista Universitaria de Investigación, vol. 3, núm. 1. Universidad Pedagógica Experimental Libertador. Venezuela.

Stahl G., Zhou n., Toledo R. (2006). The virtual math teams project: A global math discourse community. Documento presentado en: the International Conference on Computers and Education (ICCE '06), Beijing, China. Recuperado de http://idea.library.drexel. edu/bitstream/1860/1712/1/2006150046.pdf 